Bull. Korean Math. Soc. 50 (2013), No. 2, pp. 431-440

http://dx.doi.org/10.4134/BKMS.2013.50.2.431

\title{
THE FIRST POSITIVE EIGENVALUE OF THE DIRAC OPERATOR ON 3-DIMENSIONAL SASAKIAN MANIFOLDS
}

\author{
Eui Chul Kim
}

\begin{abstract}
Let $\left(M^{3}, g\right)$ be a 3-dimensional closed Sasakian spin manifold. Let $S_{\min }$ denote the minimum of the scalar curvature of $\left(M^{3}, g\right)$. Let $\lambda_{1}^{+}>0$ be the first positive eigenvalue of the Dirac operator of $\left(M^{3}, g\right)$. We proved in [13] that if $\lambda_{1}^{+}$belongs to the interval $\lambda_{1}^{+} \in\left(\frac{1}{2}, \frac{5}{2}\right)$, then $\lambda_{1}^{+}$satisfies $\lambda_{1}^{+} \geq \frac{S_{\min }+6}{8}$. In this paper, we remove the restriction "if $\lambda_{1}^{+}$belongs to the interval $\lambda_{1}^{+} \in\left(\frac{1}{2}, \frac{5}{2}\right)$ " and prove

$$
\lambda_{1}^{+} \geq \begin{cases}\frac{S_{\min }+6}{8} & \text { for } \quad-\frac{3}{2}<S_{\min } \leq 30 \\ \frac{1+\sqrt{2 S_{\min }+4}}{2} & \text { for } \quad S_{\min } \geq 30\end{cases}
$$
\end{abstract}

\section{Introduction}

Let $\left(M^{n}, g\right)$ be a closed Riemannian spin manifold. The Levi-Civita connection $\nabla$ and the Dirac operator $D$, acting on sections $\psi \in \Gamma(\Sigma(M))$ of the spinor bundle $\Sigma(M)$ over $M^{n}$, are respectively expressed as

$$
\nabla_{X} \psi=X(\psi)+\frac{1}{4} \sum_{u=1}^{n} E_{u} \cdot \nabla_{X} E_{u} \cdot \psi
$$

and

$$
D \psi=\sum_{u=1}^{n} E_{u} \cdot \nabla_{E_{u}} \psi,
$$

where $X(\psi)$ is the directional derivative of $\psi$ along a vector field $X \in \Gamma(T(M))$, $\left(E_{1}, \ldots, E_{n}\right)$ is a local orthonormal frame on $\left(M^{n}, g\right)$ and the dot "." indicates the Clifford multiplication [6]. Since $\left(M^{n}, g\right)$ is a closed manifold, the spectrum $\operatorname{Spec}(D)$ of the Dirac operator $D$ is discrete and real and will be written as

$$
\cdots \leq \lambda_{2}^{-} \leq \lambda_{1}^{-} \leq 0 \leq \lambda_{1}^{+} \leq \lambda_{2}^{+} \leq \cdots,
$$

where each eigenvalue except zero is repeated as many times as its multiplicity. The nonzero eigenvalue $\lambda_{1}^{-} \neq 0$ and $\lambda_{1}^{+} \neq 0$ are called the first negative eigenvalue and the first positive eigenvalue, respectively. The eigenvalue

Received September 19, 2011.

2010 Mathematics Subject Classification. 53C25, 53C27.

Key words and phrases. Dirac operator, eigenvalues, Sasakian manifolds. 
$\lambda_{1} \in \operatorname{Spec}(D)$ with $\left|\lambda_{1}\right|=\min \left\{\left|\lambda_{1}^{-}\right|,\left|\lambda_{1}^{+}\right|\right\}$is called the first eigenvalue. A classical result about the first Dirac eigenvalue is the Friedrich inequality

$$
\left|\lambda_{1}\right| \geq \sqrt{\frac{n S_{\min }}{4(n-1)}},
$$

where $S_{\text {min }}$ denotes the minimum of the scalar curvature [5, 9]. (1.1) holds for all closed Riemannian spin manifolds $\left(M^{n}, g\right)$ with positive scalar curvature $S>0$ and the limiting case of this inequality is characterized by the existence of a Killing spinor $\psi$,

$$
\nabla_{X} \psi=a X \cdot \psi, \quad a \in \mathbb{R} .
$$

If $\left(M^{n}, g\right)$ is of odd dimension $n, n \equiv 3 \bmod 4$, then $\operatorname{Spec}(D)$ is generally asymmetric with respect to zero $[2,11]$. In that case, a problem of interest is to find an optimal estimate for $\lambda_{1}^{-}$and that for $\lambda_{1}^{+}$, respectively $[7,13]$.

A Sasakian manifold is an odd-dimensional Riemannian manifold $\left(M^{2 m+1}\right.$, $g), m \geq 1$, equipped with a tensor field $\phi$ of type $(1,1)$, a vector field $\xi$ and a 1 -form $\eta$ that satisfy

$$
\begin{aligned}
& \eta(\xi)=1, \quad \phi^{2}(X)=-X+\eta(X) \xi, \\
& g(\phi X, \phi Y)=g(X, Y)-\eta(X) \eta(Y), \\
& \left(\nabla_{X} \phi\right)(Y)=g(X, Y) \xi-\eta(Y) X
\end{aligned}
$$

for all vector fields $X, Y \in \Gamma(\Sigma(M))$. Over Sasakian spin manifolds, a special class of spinors deserves attention.

Definition 1.1. A spinor field $\psi$ on Sasakian spin manifold $\left(M^{2 m+1}, \phi, \xi, \eta, g\right)$ is called an eta-Killing spinor with Killing pair $(a, b)$ if it satisfies

$$
\nabla_{X} \psi=a X \cdot \psi+b \eta(X) \xi \cdot \psi
$$

for some real numbers $a, b \in \mathbb{R}$ and for all vector fields $X$.

For the relations between the Killing pair $(a, b)$ of an eta-Killing spinor and the geometry of the Sasakian manifold, we refer to $[8,12]$. It turns out in Section 3 that the existence of an eta-Killing spinor characterizes the limiting case of inequalities (3.4)-(3.6).

As discussed in the introduction of [13], an observation of the Dirac spectrum of a round sphere $S^{2 m+1}$ with Berger metric gives rise to the following two questions:

Let $\left(M^{3}, \phi, \xi, \eta, g\right)$ be a 3 -dimensional closed Sasakian spin manifold.

(1) Does the first negative Dirac eigenvalue $\lambda_{1}^{-}$on $\left(M^{3}, \phi, \xi, \eta, g\right)$ satisfies

$$
\lambda_{1}^{-} \leq \frac{1-\sqrt{2 S_{\min }+4}}{2} \quad \text { for } \quad S_{\min }>-\frac{3}{2} ?
$$

(2) Does the first positive Dirac eigenvalue $\lambda_{1}^{+}$on $\left(M^{3}, \phi, \xi, \eta, g\right)$ satisfies

$$
\lambda_{1}^{+} \geq \begin{cases}\frac{S_{\min }+6}{8} & \text { for }-2<S_{\min } \leq 30, \\ \frac{1+\sqrt{2 S_{\min }+4}}{2} & \text { for } \quad S_{\min } \geq 30 ?\end{cases}
$$


Note that both (1.2) and (1.3) improve Friedrich's inequality (1.1). We proved in [13] that the answer to the first question is positive. But as to the second question, we gave only a partial answer (see Theorem 3.2 in [13]):

If the first positive Dirac eigenvalue $\lambda_{1}^{+}$belongs to the interval $\lambda_{1}^{+} \in\left(\frac{1}{2}, \frac{5}{2}\right)$, then $\lambda_{1}^{+}$satisfies $\lambda_{1}^{+} \geq \frac{S_{\min }+6}{8}$.

The aim of this paper is to give a complete answer to the second question, removing the uncomfortable restriction "if $\lambda_{1}^{+}$belongs to the interval $\lambda_{1}^{+} \epsilon$ $\left(\frac{1}{2}, \frac{5}{2}\right)$ ". Namely, we will prove Theorem 3.1. When comparing (3.10) with (1.3), there is a slight change in the scalar curvature restriction from $-2<$ $S_{\min } \leq 30$ to $-\frac{3}{2}<S_{\min } \leq 30$. It is pointed out in Remark 3.3 that the latter restriction is more reasonable.

\section{A natural deformation of the Levi-Civita connection}

To prove Proposition 3.1 in the next section, we will apply a deformation technique for spin connections. In the former part of this section we briefly review some general properties of regular Sasakian manifolds [3, 4, 14, 15], which help clarify the geometric implication of our deformation of the LeviCivita connection (see (2.9)).

A Sasakian manifold $\left(M^{2 m+1}, \phi, \xi, \eta, g\right)$ is called regular if every point of $M^{2 m+1}$ has a neighbourhood through which any integral curve of the unit vector field $\xi$ passes at most once. In that case, all the orbits of $\xi$ have the same period and $M^{2 m+1}$ turns out to be the total space of a principal $S^{1}$-bundle $\pi: M^{2 m+1} \longrightarrow N^{2 m}$. Regarding the contact form $\eta$ as a $U(1)$-connection form $\sqrt{-1} \eta$ with values in $\sqrt{-1} \mathbb{R}$, we can realize a closed 2 -form representing the first Chern class of the principal $S^{1}$-bundle $\pi: M^{2 m+1} \longrightarrow N^{2 m}$ as the curvature form

$$
c_{1}\left(M^{2 m+1} \longrightarrow N^{2 m}\right)=-\frac{1}{2 \pi}\left[\pi_{*} d \eta\right] \in H^{2}\left(N^{2 m} ; \mathbb{Z}\right) .
$$

The Sasakian structure $(\phi, \xi, \eta, g)$ on the total space $M^{2 m+1}$ then induces a Kähler structure $\left(J, g_{N}\right)$ on the base manifold $N^{2 m}$ via the relations

$$
\begin{aligned}
\pi_{*} \circ \phi & =J \circ \pi_{*}, \\
g & =\pi^{*} g_{N}+\eta \otimes \eta .
\end{aligned}
$$

As a consequence of (2.1)-(2.2), the fundamental form $\Phi=\frac{1}{2} d \eta$ on the total space $\left(M^{2 m+1}, \phi, \xi, \eta, g\right)$ coincides with the pull-back $\Phi=\pi^{*} \Omega$ of the fundamental form $\Omega$ associated to $\left(N^{2 m}, J, g_{N}\right)$. Let $\left(F_{1}, \ldots, F_{2 m}\right)$ be a local orthonormal fame on $\left(N^{2 m}, J, g_{N}\right)$ and consider its horizontal lift $\left(F_{1}^{H}, \ldots, F_{2 m}^{H}, \xi\right)$. Proceeding as in Example 6.1 of [8], we find that the Levi-Civita connection $\nabla$ of $\left(M^{2 m+1}, \phi, \xi, \eta, g\right)$ is related to that $\nabla^{N}$ of $\left(N^{2 m}, J, g_{N}\right)$ by

$$
\nabla_{F_{u}^{H}} F_{v}^{H}=\left(\nabla_{F_{u}}^{N} F_{v}\right)^{H}-\Omega_{u v} \xi
$$


where $\Omega_{u v}:=\Omega\left(F_{u}, F_{v}\right)=g_{N}\left(F_{u}, J\left(F_{v}\right)\right)$. Moreover, it holds that

$$
\nabla_{F_{v}^{H}} \xi=\nabla_{\xi} F_{v}^{H}=-\phi\left(F_{v}^{H}\right) .
$$

The Ricci tensor Ric and scalar curvature $S$ of $\left(M^{2 m+1}, \phi, \xi, \eta, g\right)$ are related to those $\operatorname{Ric}_{N}, S_{N}$ of $\left(N^{2 m}, J, g_{N}\right)$ by

$$
\operatorname{Ric}\left(W_{1}^{H}, W_{2}^{H}\right)=\operatorname{Ric}_{N}\left(W_{1}, W_{2}\right)-2 g_{N}\left(W_{1}, W_{2}\right), \quad W_{1}, W_{2} \in \Gamma(T(N)),
$$

and

$$
S=S_{N}-2 m,
$$

respectively. Let us assume that $\left(N^{2 m}, J, g_{N}\right)$ is a spin manifold and $\left(M^{2 m+1}\right.$, $\phi, \xi, \eta, g)$ is equipped with a spin structure obtained by pull-back from $\left(N^{2 m}, J\right.$, $\left.g_{N}\right)$. Let $\varphi$ be a spinor field on $N^{2 m}$ and $\varphi^{H}$ be its horizontal lift. Then the spinor derivative $\nabla \varphi^{H}$ on $\left(M^{2 m+1}, \phi, \xi, \eta, g\right)$ is related to that $\nabla^{N} \varphi$ on $\left(N^{2 m}, J, g_{N}\right)$ by

$$
\begin{aligned}
\nabla_{W H} \varphi^{H} & =\left(\nabla_{W}^{N} \varphi\right)^{H}+\frac{1}{2} \phi\left(W^{H}\right) \cdot \xi \cdot \varphi^{H}, \quad W \in \Gamma(T(N)), \\
\nabla_{\xi} \varphi^{H} & =\frac{1}{2} \Phi \cdot \varphi^{H} .
\end{aligned}
$$

A spinor field $\psi$ on $M^{2 m+1}$ is projectable onto $N^{2 m}$, i.e., there exists some spinor field $\varphi$ on $N^{2 m}$ with $\psi=\varphi^{H}$ if the directional derivative $\xi(\psi)$ vanishes identically. From (2.4), it follows that $\psi \in \Gamma(\Sigma(M))$ is projectable if and only if $\nabla_{\xi} \psi=\frac{1}{2} \Phi \cdot \psi$.

Let $\left(M^{2 m+1}, \phi, \xi, \eta, g\right)$ be a (possibly irregular) Sasakian spin manifold. Let $\xi^{\perp}$ denote the orthogonal complement of the vector field $\xi$ in the tangent bundle $T(M)$. We deform the Levi-Civita connection $\nabla$ in the subbundle $\xi^{\perp} \subset T(M)$,

$$
\bar{\nabla}_{V} \psi=\nabla_{V} \psi-\frac{1}{2} \phi(V) \cdot \xi \cdot \psi, \quad V \in \Gamma\left(\xi^{\perp}\right), \quad \psi \in \Gamma(\Sigma(M)) .
$$

The deformed connection $\bar{\nabla}_{V}$ has a remarkable property in that it commutes with the fundamental form $\Phi$

$$
\bar{\nabla}_{V} \circ \Phi=\Phi \circ \bar{\nabla}_{V}
$$

For more interesting information about the connection $\bar{\nabla}_{V}$, we refer to [1]. Define first-order operators $\bar{C}, \bar{Q}$ acting on spinor fields $\psi \in \Gamma(\Sigma(M))$ by

$$
\begin{aligned}
& \bar{C} \psi=\sum_{u=1}^{2 m} E_{u} \cdot \bar{\nabla}_{E_{u}} \psi, \\
& \bar{Q} \psi=\sum_{u=1}^{2 m} \phi\left(E_{u}\right) \cdot \bar{\nabla}_{E_{u}} \psi,
\end{aligned}
$$

where $\left(E_{1}, \ldots, E_{2 m}, \xi\right)$ is a local orthonormal frame on $\left(M^{2 m+1}, \phi, \xi, \eta, g\right)$. Both $\bar{C}$ and $\bar{Q}$ are self-adjoint with respect to the $L^{2}$-Hermitian product. But neither $\bar{C}$ nor $\bar{Q}$ is elliptic. 
Suppose that $\left(M^{2 m+1}, \phi, \xi, \eta, g\right)$ is regular, i.e., it is the total space of a circle bundle $M^{2 m+1} \longrightarrow N^{2 m}$ and the spin structure of $M^{2 m+1}$ is obtained by pull-back from $N^{2 m}$. Let $D_{N}$ be the Dirac operator of $\left(N^{2 m}, J, g_{N}\right)$ and let $\widetilde{D}_{N}$ be the $J$-twist of $D_{N}$ defined by

$$
\widetilde{D}_{N} \varphi=\sum_{u=1}^{2 m} J\left(F_{u}\right) \cdot \nabla_{F_{u}}^{N} \varphi
$$

Then, from (2.7) we see that $\bar{\nabla}_{W^{H}}, \bar{C}, \bar{Q}$ coincide with the pull-back of $\nabla_{W}^{N}$, $D_{N}, \widetilde{D}_{N}$, respectively. Inspired by this correspondence, we define a Sasakian analogue of the Kählerian twistor spinors [10].

Definition 2.1. A spinor field $\psi \in \Gamma(\Sigma(M))$ on Sasakian spin manifold $\left(M^{2 m+1}\right.$, $\phi, \xi, \eta, g)$ is called a Sasakian twistor spinor of type $(a, b)$ if

$$
\bar{\nabla}_{V} \psi=a V \cdot \bar{C} \psi+b \phi(V) \cdot \bar{Q} \psi
$$

holds for some numbers $a, b \in \mathbb{R}$ and for all vector fields $V \in \Gamma\left(\xi^{\perp}\right)$ orthogonal to $\xi$.

Example 2.1. Using Lemma 3.2 and Proposition 3.1 in [12], one verifies that, on a Sasakian spin manifold of dimension $2 m+1 \geq 5$, any eta-Killing spinor with Killing pair $(a, b), a \neq 0, b \neq 0$, is a Sasakian twistor spinor of type $(0,0)$.

We shall see in the next section that, on a 3-dimensional closed Sasakian spin manifold, the existence of a Sasakian twistor spinor characterizes the limiting case of inequalities (3.4)-(3.6).

We close the section with three lemmata that we will need in the next section. To state Lemma 2.3 we use the notation $()=,\operatorname{Re}\langle$,$\rangle denoting the real part of$ the standard Hermitian product $\langle$,$\rangle on the spinor bundle \Sigma(M)$ over $M^{2 m+1}$.

Lemma 2.1. On a Sasakian spin manifold $\left(M^{2 m+1}, \phi, \xi, \eta, g\right)$, the operator identity

$$
\bar{C}^{2}=D^{2}+\nabla_{\xi} \nabla_{\xi}-\xi \circ \bar{Q}-2 \Phi \circ \nabla_{\xi}+\Phi \circ \Phi
$$

holds.

Lemma 2.2. Let $\left(M^{2 m+1}, \phi, \xi, \eta, g\right)$ be a closed Sasakian spin manifold. Let $\left(E_{1}, \ldots, E_{2 m}, \xi\right)$ be a local orthonormal frame on $M^{2 m+1}$ and let $\bar{\nabla}_{E_{u}}^{*}$ denote the adjoint of $\bar{\nabla}_{E_{u}}$ with respect to the $L^{2}$-Hermitain product. Then we have

$$
\sum_{u=1}^{2 m} \bar{\nabla}_{E_{u}}^{*} \bar{\nabla}_{E_{u}}=\bar{C}^{2}-\frac{1}{4} S+2 \Phi \circ \nabla_{\xi}-\Phi \circ \Phi-\frac{m}{2} .
$$

Lemma $2.3([13])$. Let $\left(M^{2 m+1}, \phi, \xi, \eta, g\right)$ be a closed Sasakian spin manifold and let $\mu$ denote the volume form. Then, for any eigenspinor $\psi$ of the Dirac operator $D$ with eigenvalue $\lambda$, we have

$$
0=\int_{M^{2 m+1}}\left[2\left(\nabla_{\xi} \psi, \nabla_{\xi} \psi\right)-3\left(\nabla_{\xi} \psi, \Phi \cdot \psi\right)+2 \lambda\left(\nabla_{\xi} \psi, \xi \cdot \psi\right)\right.
$$




$$
-\lambda(\Phi \cdot \psi, \xi \cdot \psi)+(\Phi \cdot \psi, \Phi \cdot \psi)] \mu .
$$

\section{Estimates of small Dirac eigenvalues on 3-dimensional Sasakian} manifolds revisited

Let us realize the three-dimensional Clifford algebra using the matrices

$$
E_{1}=\left(\begin{array}{cc}
\sqrt{-1} & 0 \\
0 & -\sqrt{-1}
\end{array}\right), \quad E_{2}=\left(\begin{array}{cc}
0 & \sqrt{-1} \\
\sqrt{-1} & 0
\end{array}\right), \quad E_{3}=\left(\begin{array}{cc}
0 & 1 \\
-1 & 0
\end{array}\right) .
$$

Then the relations

$$
E_{1} \cdot E_{2}=-E_{3}, \quad E_{2} \cdot E_{3}=-E_{1}, \quad E_{3} \cdot E_{1}=-E_{2}
$$

are valid. It follows that, on a 3 -dimensional closed Sasakian spin manifold $\left(M^{3}, \phi, \xi, \eta, g\right)$, the operator identities

$$
\Phi=\xi, \quad \xi \cdot \bar{Q}=D-\xi \cdot \nabla_{\xi}-1
$$

hold and hence (2.10)-(2.12) simplify to

$$
\bar{C}^{2}=D^{2}+\nabla_{\xi} \nabla_{\xi}-D-\xi \cdot \nabla_{\xi}
$$

and

$$
\sum_{u=1}^{2} \bar{\nabla}_{E_{u}}^{*} \bar{\nabla}_{E_{u}}=D^{2}-\frac{1}{4} S+\nabla_{\xi} \nabla_{\xi}-D+\xi \cdot \nabla_{\xi}+\frac{1}{2}
$$

and

$$
0=\int_{M^{3}}\left[2\left(\nabla_{\xi} \psi, \nabla_{\xi} \psi\right)+(2 \lambda-3)\left(\nabla_{\xi} \psi, \xi \cdot \psi\right)+(1-\lambda)(\psi, \psi)\right] \mu,
$$

respectively.

Proposition 3.1. Let $\left(M^{3}, \phi, \xi, \eta, g\right)$ be a 3-dimensional closed Sasakian spin manifold and suppose that the scalar curvature satisfies $S_{\min }>-2$. Let $\lambda$ be an eigenvalue of the Dirac operator $D$.

(i) If $\lambda<\frac{1}{2}$, then the inequality

$$
\lambda \leq \frac{1-\sqrt{2 S_{\min }+4}}{2}
$$

holds. The limiting case of (3.4) occurs if and only if the scalar curvature is constant and there exists an eta-Killing spinor with Killing pair $\left(\frac{-2+\sqrt{2 S+4}}{4}, \frac{4-\sqrt{2 S+4}}{4}\right)$.

(ii) If $\frac{1}{2}<\lambda \leq \frac{9}{2}$, then the inequality

$$
\lambda \geq \frac{S_{\min }+6}{8}
$$

holds. The limiting case of (3.5) occurs if and only if the scalar curvature is constant and there exists an eta-Killing spinor with Killing pair $\left(-\frac{1}{2},-\frac{S}{8}+\frac{3}{4}\right)$.

(iii) If $\lambda \geq \frac{9}{2}$, then the inequality

$$
\lambda \geq \frac{1+\sqrt{2 S_{\min }+4}}{2}
$$


holds. The limiting case of (3.6) occurs if and only if the scalar curvature is constant and there exists an eta-Killing spinor with Killing pair $\left(\frac{-2-\sqrt{2 S+4}}{4}, \frac{4+\sqrt{2 S+4}}{4}\right)$.

Proof. Let $\left(E_{1}, E_{2}=\phi\left(E_{1}\right), \xi\right)$ be an adapted local orthonormal frame on $\left(M^{3}, \phi, \xi, \eta, g\right)$. Let $\psi$ be an eigenspinor of $D$ with eigenvalue $\lambda$. Introducing free parameters $\kappa, \tau \in \mathbb{R}$ to control the unnecessary terms, we compute

$$
\begin{aligned}
H= & \sum_{u=1}^{2} \int_{M^{3}}\left(\bar{\nabla}_{E_{u}} \psi+\frac{1}{2} E_{u} \cdot \bar{C} \psi, \bar{\nabla}_{E_{u}} \psi+\frac{1}{2} E_{u} \cdot \bar{C} \psi\right) \mu \\
& +\kappa^{2} \int_{M^{3}}\left(\nabla_{\xi} \psi-\tau \xi \cdot \psi, \nabla_{\xi} \psi-\tau \xi \cdot \psi\right) \mu \\
= & \sum_{u=1}^{2} \int_{M^{3}}\left(\bar{\nabla}_{E_{u}} \psi, \bar{\nabla}_{E_{u}} \psi\right) \mu-\frac{1}{2} \int_{M^{3}}\left(\bar{C}^{2} \psi, \psi\right) \mu \\
& +\int_{M^{3}}\left[\kappa^{2}\left(\nabla_{\xi} \psi, \nabla_{\xi} \psi\right)-2 \kappa^{2} \tau\left(\nabla_{\xi} \psi, \xi \cdot \psi\right)+\kappa^{2} \tau^{2}(\psi, \psi)\right] \mu .
\end{aligned}
$$

We apply (3.1)-(3.2) to obtain

$$
\begin{aligned}
H= & \int_{M^{3}}\left(\frac{\lambda^{2}}{2}-\frac{\lambda}{2}+\frac{1}{2}-\frac{S}{4}+\kappa^{2} \tau^{2}\right)(\psi, \psi) \mu \\
& +\int_{M^{3}}\left[\left(\kappa^{2}-\frac{1}{2}\right)\left(\nabla_{\xi} \psi, \nabla_{\xi} \psi\right)-\left(2 \kappa^{2} \tau+\frac{3}{2}\right)\left(\nabla_{\xi} \psi, \xi \cdot \psi\right)\right] \mu .
\end{aligned}
$$

Due to (3.3) we have

$$
\begin{aligned}
H= & \int_{M^{3}}\left[\frac{\lambda^{2}}{2}-\frac{\lambda}{2}+\frac{1}{2}-\frac{S}{4}+\kappa^{2} \tau^{2}+\left(\kappa^{2}-\frac{1}{2}\right)\left(\frac{\lambda}{2}-\frac{1}{2}\right)\right](\psi, \psi) \mu \\
& -\int_{M^{3}}\left[\left(\kappa^{2}-\frac{1}{2}\right)\left(\lambda-\frac{3}{2}\right)+2 \kappa^{2} \tau+\frac{3}{2}\right]\left(\nabla_{\xi} \psi, \xi \cdot \psi\right) \mu
\end{aligned}
$$

Let us now choose

$$
\kappa^{2}=\frac{2 \lambda-9}{2(2 \lambda-3+4 \tau)} \geq 0 .
$$

Then the latter integral of (3.7) vanishes and we obtain

$$
H=\frac{1}{4} \int_{M^{3}}\left[2 \lambda^{2}-2 \lambda+2-S+4 \kappa^{2} \tau^{2}+\left(2 \kappa^{2}-1\right)(\lambda-1)\right](\psi, \psi) \mu \geq 0 .
$$

The proof idea for Theorem 3.1 and that for Theorem 3.2 in [13] suggest us to consider the case $\tau=\frac{1}{2}$ and $\tau=1-\lambda$, respectively. In case of $\tau=\frac{1}{2}$, we have

$$
\kappa^{2}=\frac{2 \lambda-9}{2(2 \lambda-1)}
$$


and obtain

$$
H=\frac{1}{4} \int_{M^{3}}\left[2 \lambda^{2}-2 \lambda-S-\frac{3}{2}\right](\psi, \psi) \mu,
$$

which proves part (i) as well as part (iii) of the proposition. The limiting case of part (i) occurs if and only if there exists a solution to the system of equations

$$
\begin{gathered}
\bar{\nabla}_{V} \psi+\frac{1}{2} V \cdot \bar{C} \psi=0, \quad \nabla_{\xi} \psi=\frac{1}{2} \xi \cdot \psi, \quad V \in \xi^{\perp} \\
\Longleftrightarrow \quad \nabla_{V} \psi=-\left(\frac{\lambda}{2}+\frac{1}{4}\right) V \cdot \psi, \quad \nabla_{\xi} \psi=\frac{1}{2} \xi \cdot \psi, \quad V \in \xi^{\perp},
\end{gathered}
$$

which is equivalent to the condition that the scalar curvature is constant and there exists an eta-Killing spinor with Killing pair $\left(\frac{-2+\sqrt{2 S+4}}{4}, \frac{4-\sqrt{2 S+4}}{4}\right)$. In the same way, one verifies the condition for the limiting case of part (iii). Let us now consider the other case $\tau=1-\lambda$. In that case, we have

$$
\kappa^{2}=-\frac{2 \lambda-9}{2(2 \lambda-1)}
$$

and obtain

$$
H=\frac{1}{4} \int_{M^{3}}(8 \lambda-S-6)(\psi, \psi) \mu,
$$

which proves part (ii) of the proposition. The condition for the limiting case of part (ii) is easy to check.

Remark 3.1. Any eta-Killing spinor with Killing pair $\left(-\frac{1}{2},-\frac{S}{8}+\frac{3}{4}\right)$ is a Sasakian twistor spinor of type $(0,0)$. Any eta-Killing spinor with Killing pair $\left(\frac{-2 \pm \sqrt{2 S+4}}{4}, \frac{4 \mp \sqrt{2 S+4}}{4}\right)$ is a Sasakian twistor spinor of type $\left(-\frac{1}{2}, 0\right)$.

Remark 3.2. Suppose that $\left(M^{3}, \phi, \xi, \eta, g\right)$ is a circle bundle $\pi: M^{3} \longrightarrow N^{2}$ and admits an eta-Killing spinor $\psi_{1}$ with Killing pair $\left(\frac{-2+\sqrt{2 S+4}}{4}, \frac{4-\sqrt{2 S+4}}{4}\right)$ as well as an eta-Killing spinor $\psi_{2}$ with Killing pair $\left(\frac{-2-\sqrt{2 S+4}}{4}, \frac{4+\sqrt{2 S+4}}{4}\right)$. Then, due to (3.9), both $\psi_{1}$ and $\psi_{2}$ are projectable onto $N^{2}$ and there exist Killing spinors $\varphi_{1}, \varphi_{2}$ on $N^{2}$ with $\psi_{k}=\pi^{*} \varphi_{k}, k=1,2$. The base 2-manifold $N^{2}$ is in fact isometric to a sphere with constant scalar curvature $S+2$ (see $(2.6))$ and $\varphi_{1}, \varphi_{2}$ satisfy

$$
\begin{aligned}
& \nabla_{W}^{N} \varphi_{1}=\frac{1}{4} \sqrt{2(S+2)} W \cdot \varphi_{1} \\
& \nabla_{W}^{N} \varphi_{2}=-\frac{1}{4} \sqrt{2(S+2)} W \cdot \varphi_{2}, \quad W \in \Gamma(T(N)) .
\end{aligned}
$$

Proposition 3.2. Let $\left(M^{3}, \phi, \xi, \eta, g\right)$ be a 3-dimensional closed Sasakian spin manifold. Then there exists an eigenspinor $\psi$ of the Dirac operator $D$ with eigenvalue $\lambda=\frac{1}{2}$ only if the minimum of the scalar curvature satisfies $S_{\min } \leq$ -2 . 
Proof. We see from (3.1) that

$$
\begin{aligned}
& \int_{M^{3}}\left[(\bar{C} \varphi, \bar{C} \varphi)+\left(\nabla_{\xi} \varphi-\frac{1}{2} \xi \cdot \varphi, \nabla_{\xi} \varphi-\frac{1}{2} \xi \cdot \varphi\right)\right] \mu \\
= & \int_{M^{3}}\left(D \varphi-\frac{1}{2} \varphi, D \varphi-\frac{1}{2} \varphi\right) \mu
\end{aligned}
$$

holds for any spinor field $\varphi$ on $M^{3}$. Thus, if there exists an eigenspinor $\psi$ of $D$ with eigenvalue $\lambda=\frac{1}{2}$, then we have

$$
\bar{C} \psi=0, \quad \nabla_{\xi} \psi=\frac{1}{2} \xi \cdot \psi
$$

and so

$$
\begin{aligned}
& \sum_{i=1}^{2} \int_{M^{3}}\left(\bar{\nabla}_{E_{i}} \psi, \bar{\nabla}_{E_{i}} \psi\right) \mu \\
= & \int_{M^{3}}\left[(\bar{C} \psi, \bar{C} \psi)-\frac{S}{4}(\psi, \psi)-2\left(\nabla_{\xi} \psi, \xi \cdot \psi\right)+\frac{1}{2}(\psi, \psi)\right] \mu \\
= & \int_{M^{3}}\left(-\frac{1}{4} S-\frac{1}{2}\right)(\psi, \psi) \mu,
\end{aligned}
$$

which yields $S_{\min } \leq-2$.

Theorem 3.1. Let $\left(M^{3}, \phi, \xi, \eta, g\right)$ be a 3-dimensional closed Sasakian spin manifold. Then the first positive eigenvalue $\lambda_{1}^{+}$of the Dirac operator $D$ satisfies

$$
\lambda_{1}^{+} \geq \begin{cases}\frac{S_{\min }+6}{8} & \text { for }-\frac{3}{2}<S_{\min } \leq 30 \\ \frac{1+\sqrt{2 S_{\min }+4}}{2} & \text { for } S_{\min } \geq 30 .\end{cases}
$$

The limiting case of (3.10) occurs if and only if the scalar curvature is constant and there exists an eta-Killing spinor with Killing pair

$$
\begin{cases}\left(-\frac{1}{2},-\frac{S}{8}+\frac{3}{4}\right) & \text { for }-\frac{3}{2}<S_{\min } \leq 30 \\ \left(\frac{-2-\sqrt{2 S+4}}{4}, \frac{4+\sqrt{2 S+4}}{4}\right) & \text { for } \quad S_{\min } \geq 30\end{cases}
$$

Proof. Because of the restriction $S_{\min }>-\frac{3}{2}$, it follows from Proposition 3.2 and part (i) of Proposition 3.1 that $\lambda_{1}^{+} \leq \frac{1}{2}$ is not allowed. Consequently, part (ii) and (iii) of Proposition 3.1 together give

$$
\lambda_{1}^{+} \geq \min \left\{\frac{S_{\min }+6}{8}, \frac{1+\sqrt{2 S_{\min }+4}}{2}\right\},
$$

which we can equivalently rewrite as (3.10). The condition for the limiting case of (3.10) is easy to check.

Remark 3.3. Let $\left(M^{3}, \phi, \xi, \eta, g\right)$ be a simply-connected Sasakian spin manifold of dimension 3 and suppose that the scalar curvature $S$ is constant. We proved in [8] that if $S \geq-2$, then there exists an eta-Killing spinor $\psi$ with Killing 
pair $\left(\frac{-2+\sqrt{2 S+4}}{4}, \frac{4-\sqrt{2 S+4}}{4}\right)$. In particular, if we choose $S=-\frac{3}{2}$, then $\psi$ is a harmonic spinor. This means that our restriction $S_{\min }>-\frac{3}{2}$ in Theorem 3.1 is reasonable.

Acknowledgements. This work was supported by a grant from 2010 Research Fund of Andong National University.

\section{References}

[1] I. Agricola, The Srni lectures on non-integrable geometries with torsion, Arch. Math. (Brno) 42 (2006), 5-84.

[2] M. F. Atiyah, V. K. Patodi, and I. M. Singer, Spectral asymmetry and Riemannian geometry. I, Math. Proc. Cambridge Philos. Soc. 77 (1975), 43-69.

[3] F. A. Belgun, Normal CR structure on compact 3-manifolds, Math. Z. 238 (2001), no. 3, 441-460.

[4] D. E. Blair, Riemannian Geometry of Contact and Symplectic Manifolds, Birkhäuser, Boston/Basel/Berlin, 2002.

[5] Th. Friedrich, Der erste Eigenwert des Dirac-Operators einer kompakten Riemannschen Mannigfaltigkeit nichtnegativer Skalarkrümmung, Math. Nachr. 97 (1980), 117-146.

[6] Dirac Operators in Riemannian Geometry, Graduate Studies in Mathematics, vol. 25, AMS, Providence, RI, 2000.

7] - The second Dirac eigenvalue of a nearly parallel $G_{2}$-manifold, Adv. Appl. Clifford Algebr. 22 (2012), no. 2, 301-311.

[8] Th. Friedrich and E. C. Kim, The Einstein-Dirac equation on Riemannian spin manifolds, J. Geom. Phys. 33 (2000), no. 1-2, 128-172.

[9] N. Ginoux, The Dirac Spectrum, Lecture Notes in Mathematics, Springer-Verlag, Berlin/Heidelberg, 2009.

[10] O. Hijazi, Eigenvalues of the Dirac operator on compact Kähler manifolds, Comm. Math. Phys. 160 (1994), no. 3, 563-579.

[11] E. C. Kim, The Â-genus and symmetry of the Dirac spectrum on Riemannian product manifolds, Differential Geom. Appl. 25 (2007), no. 3, 309-321.

[12] _ Dirac eigenvalues estimates in terms of divergencefree symmetric tensors, Bull. Korean Math. Soc. 46 (2009), no. 5, 949-966.

[13] _ Estimates of small Dirac eigenvalues on 3-dimensional Sasakian manifolds, Differential Geom. Appl. 28 (2010), no. 6, 648-655.

[14] S. Kobayashi, Principal fibre bundles with the 1-dimensional toroidal group, Tohoku Math. J. 8 (1956), 29-45.

[15] A. Moroianu, Opérateur de Dirac et submersions riemanniennes, Thesis, École Polytechnique, 1996.

Department of Mathematics

College of Education

ANDONG NATIONAL UNIVERSITY

Andong 760-749, KoreA

E-mail address: eckim@andong.ac.kr 